\title{
A versatile microreactor platform featuring a chemical-resistant microvalve array for addressable multiplex syntheses and assays
}

\author{
Zhishan Hua $^{1}$, Yongmei Xia ${ }^{1}$, Onnop Srivannavit ${ }^{1}$, \\ Jean-Marie Rouillard ${ }^{1}$, Xiaochuan Zhou ${ }^{2}$, Xiaolian Gao $^{3}$ \\ and Erdogan Gulari ${ }^{1}$ \\ ${ }^{1}$ Department of Chemical Engineering, The University of Michigan, Ann Arbor, \\ MI 48109-2136, USA \\ ${ }^{2}$ Atactic Technologies, Houston, TX 77054, USA \\ ${ }^{3}$ Department of Chemistry, University of Houston, Houston, TX 77004-5003, USA \\ E-mail: gulari@umich.edu
}

Received 2 February 2006, in final form 2 May 2006

Published 8 June 2006

Online at stacks.iop.org/JMM/16/1433

\begin{abstract}
A versatile microreactor platform featuring a novel chemical-resistant microvalve array has been developed using combined silicon/polymer micromachining and a special polymer membrane transfer process. The basic valve unit in the array has a typical 'transistor' structure and a PDMS/parylene double-layer valve membrane. A robust multiplexing algorithm is also proposed for individual addressing of a large array using a minimal number of signal inputs. The in-channel microvalve is leakproof upon pneumatic actuation. In open status it introduces small impedance to the fluidic flow, and allows a significantly larger dynamic range of flow rates

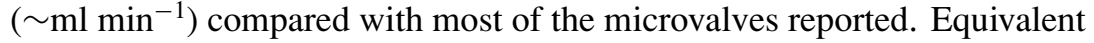
electronic circuits were established by modeling the microvalves as PMOS transistors and the fluidic channels as simple resistors to provide theoretical prediction of the device fluidic behavior. The presented microvalve/reactor array showed excellent chemical compatibility in the tests with several typical aggressive chemicals including those seriously degrading PDMS-based microfluidic devices. Combined with the multiplexing strategy, this versatile array platform can find a variety of lab-on-a-chip applications such as addressable multiplex biochemical synthesis/assays, and is particularly suitable for those requiring tough chemicals, large flow rates and/or high-throughput parallel processing. As an example, the device performance was examined through the addressed synthesis of 30-mer DNA oligonucleotides followed by sequence validation using on-chip hybridization. The results showed leakage-free valve array addressing and proper synthesis in target reactors, as well as uniform flow distribution and excellent regional reaction selectivity.
\end{abstract}

(Some figures in this article are in colour only in the electronic version) 


\section{Introduction}

The recently emerged lab-on-a-chip technology has created a strong demand for reliable and robust microvalves as the fundamental component for fluidic manipulation. The critical role of microvalves in microfluidic systems can be considered analogous to that of transistors in microelectronics circuits [1]. Also, similar to the development of microprocessors and memory chips which are essentially the large-scale integration of single transistors, there is a growing interest in the microfluidic integration of single microvalves. The resulting microvalve array will allow us to perform even more advanced and complex chemical and biomedical tasks in a miniaturized scale. For example, if microvalves are used to address an array of microreactors and deliver specific reagents to each of them, a large number of distinct reactions can be simultaneously conducted on a microchip, which will result in a revolutionary reduction in processing time and reagent costs for multiplex assays such as large-scale screening and discovery compared with traditional approaches. This microvalve/reactor array integration also provides a potential tool of parallel combinatorial synthesis to rapidly produce a library of various compounds, which can find instant use or be further assembled into valuable synthetic biomacromolecules.

As the basic element of an integrated array, the single microvalve needs to be very compact and reliable. Over the past decade, a large variety of microvalves with different structures and operation mechanisms have been developed. Among them pneumatic-actuated PDMS microvalves have become particularly popular due to the ease of fabrication and operation $[2,3]$. The extreme softness and flexibility of PDMS elastomer (Young's modulus $\sim 360-870 \mathrm{kPa}$ ) allows a highly miniaturized valve size, and large-scale microvalve integration thus becomes practical [4, 5]. However, PDMS exhibits swelling behavior in many organic solvents such as tetrahydrofuran, toluene and dichloromethane. The flow of these solvents can be impeded or even blocked in PDMS structures [6]. This limits the use of PDMS microvalves in applications involving aggressive chemicals such as many organic syntheses. In addition, the sample evaporation due to the permeability of PDMS and nonspecific adsorption of some biomacromolecules on PDMS [7] can be problematic in some applications.

Another important polymer material used for microvalves is parylene thin film, which has sufficient flexibility (Young's modulus $\sim 2.8 \mathrm{GPa}$ ) and excellent chemical resistance against most solvents, acids, bases and oxidizers. Parylene also has extremely low permeability to moisture and excellent biocompatibility [7]. Recently a number of passive valves [8] and active valves [9, 10] based on parylene have been developed using parylene surface micromachining processes. These devices have great chemical and biological compatibility compared with PDMS devices. However, the removal of the sacrificial photoresist involved in surface micromachining is a diffusion-limited and particularly time-consuming step for long channel fabrication [11]. Additionally, the vertical dimension of the surface micromachined structures is limited by the thickness of the sacrificial photoresist, which normally does not exceed $20 \mu \mathrm{m}$. The resulting microchannels therefore have a relatively limited
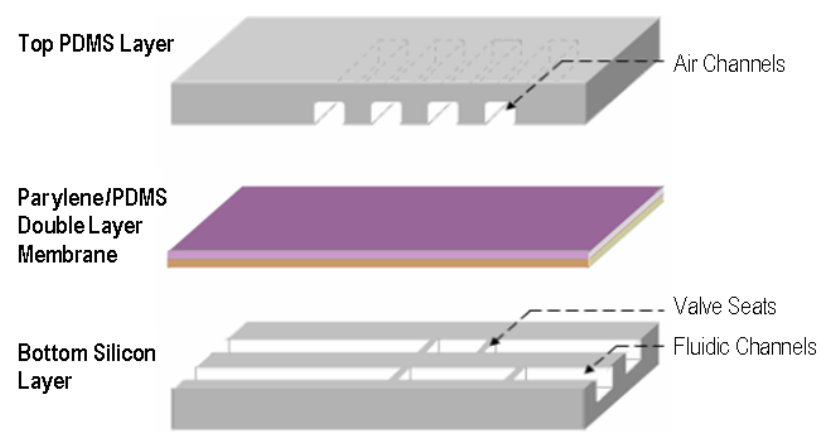

Figure 1. Exploded view of the microvalve/reactor array structure.

flow handling capacity and are not suitable for special applications where large flow rates and high throughputs are required.

A versatile and robust microfluidic platform that supports a broad spectrum of chemical and biomedical applications therefore appears promising. In this work we first developed a novel pneumatic microvalve array that employs a silicon/PDMS hybrid structure and a parylene/PDMS doublelayer valve membrane. This design combined the advantages of PDMS processing and parylene materials, and achieved many desirable device features such as ease of fabrication and tubing connection, minimum flow impedance, as well as excellent chemical resistance and biocompatibility. All of these make the microvalve particularly suitable for the handling of a wide dynamic range of flow rates as well as tough samples such as aggressive chemicals, viscous fluids, large cells and so on. The valve array was further integrated with a microreactor array into a microchip platform for multiplex syntheses and assays using a highly efficient array multiplexing strategy we proposed. Finally, addressed synthesis of DNA oligonucleotides was conducted to examine the performance of the integrated array platform.

\section{Design and theory}

\subsection{Microvalve/reactor array structure}

As schematically shown in figure 1 , the device consists of three pieces. A parylene/PDMS double-layer thin film is sandwiched between a top PDMS and bottom silicon substrate. The deep trenches on top PDMS serve as the air pressure supply lines. Fluidic channels, microreactors and valve seats are located on the bottom silicon. The upper surface of this silicon substrate is also covered by parylene thin film. All three pieces are irreversibly bonded together. As an illustration only two reactors from the entire array and their addressing microvalves are shown in figure 1.

The basic microvalve operation and addressing principle is depicted in figure 2. The individual microvalve has a typical 'transistor' configuration including an air chamber, a flexible membrane and a valve seat. This structure has proved to be very reliable and easy to implement $[1,3]$. Each microreactor is assigned a few valve units located in specific positions in the fluidic channel where the air channels on the top PDMS layer cross over, and the number of valve units for each reactor is less than that of the air channels (figure 2(a)). 


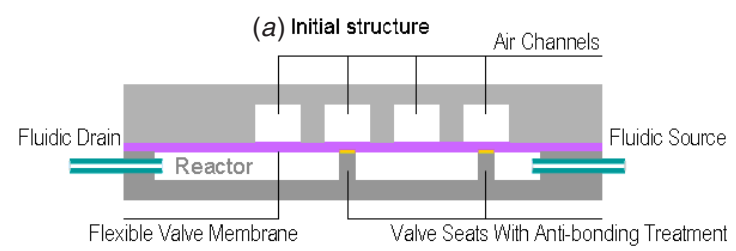

(b) All addressing valves open; reactor addressed

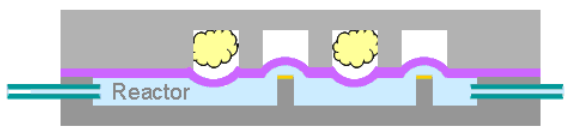

(c) Any addressing valve closes; reactor unaddressed

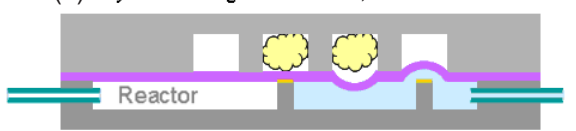

Figure 2. (a) Schematic of a microreactor cell and multiple addressing valves. (b) Opened (addressed) microreactor. (c) Closed (unaddressed) microreactor.

To address the target reactor in the array, air pressure is sent to some of the air channels while other air channels remain unpressurized or vacuumed. For a certain reactor, if all its valve seats are under these unpressurized air channels, the flexible parylene membrane over the valve seats will be lifted up by the hydraulic pressure of injected fluidic flow and generates a gap which allows the flow to pass through and enter the reactor (figure $2(b)$ ). We say that this reactor is opened or addressed. However, if any of the valve seats is under a pressurized channel, the parylene valve membrane there will be pushed down to cover the flat valve seat on bottom silicon tightly and forms a barrier blocking the fluidic flow. The reactor is thus closed or 'not addressed' (figure 2(c)). The reason for using multiple microvalve units to control each reactor will be explained in the following section.

\subsection{Array addressing strategy}

For individual addressing of a microreactor array, the most straightforward way is to assign each reactor one valve and one individual air pressure supply. However, this configuration becomes impractical as the number of reactors increases to a large value because the pressure supply channels are relatively space consuming and the demand of a large number of external solenoid valves for individual pressure inputs is usually not feasible.

Our approach to solve this issue is illustrated in figure 3, which is the design layout of a prototype microreactor array platform. The device contains 16 parallel reaction chambers that share a common flow inlet and outlet. Fluidic channels with a binary tree structure are used to uniformly distribute the fluidic flow to each of the reactors because all the reactors are geometrically identical with respect to the inlet in this way. The microvalve units for individual addressing of the 16 reactors are located in the fluidic channels next to the reactors. All the valves are distributed in a 16-row $\times$ sixcolumn array. Each row (channel) has six spots (defined by column coordinates 1 to 6 ) and three of them are occupied by microvalve units. The combination of these three column

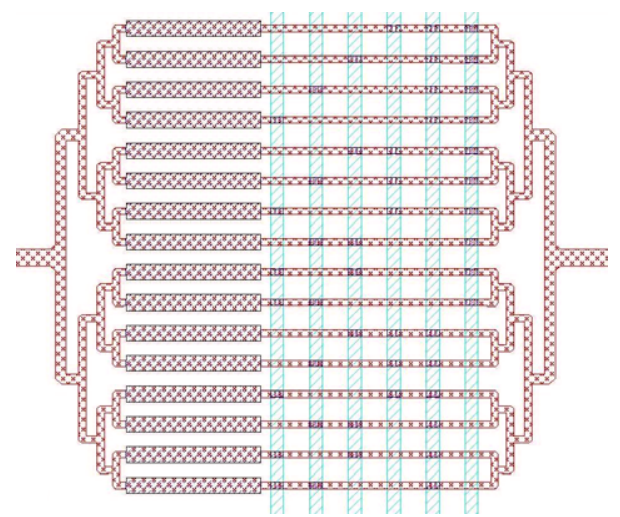

Figure 3. Layout of a prototype multiplex reaction platform.

coordinates is different from row to row. In the 16-reactor system shown in figure 3 , we used a configuration of $(1,2,3)$; $(1,2,4) ;(1,2,5) ;(1,2,6) ;(1,3,4) ;(1,3,5) ;(1,3,6)$; $(1,4,5) ;(1,4,6) ;(1,5,6),(2,3,4),(2,3,5),(2,3,6),(2,4,5)$, $(2,4,6),(2,5,6)$ for 16 rows respectively. All the valves with the same column coordinates, no matter which row they are located, will be opened or closed together because they share a common air supply channel, and a fluidic channel will be blocked if any of the three valves in it are closed. Therefore, applying pressure to any three of the six air channels will block 15 of the 16 fluidic channels and leave only one channel open. In other words, removing pressure from any three of the six initially pressurized air channels will open a certain fluidic channel as well as the microreactor connected to it. For example, releasing pressure from air channels 1, 2 and 3 (air channels 4, 5, 6 remain pressurized) opens all the valves with column coordinates 1,2 or 3 ; however only the top fluidic channel will be opened because all three valves in it are open. The other 15 fluidic channels still have at least one valve closed and thus remain blocked.

It is straightforward to determine how many air channels are required for individual addressing of a given number of microreactors. We know the number of ways, $P$, to place $M$ objects in $N$ spots ( $N$ is an even number) can be calculated using the following equation:

$$
P=N ! /[M !(N-M) !] .
$$

$P$ reaches its maximum value when

$$
M=[N / 2]
$$

and

$$
P_{\max }=N ! /[(N / 2) !]^{2} .
$$

The power of this multiplexing algorithm becomes obvious when the array size increases. For example, 16 air channels can address up to $8 ! /(4 ! 4 !)=12870$ reactors. This is even more efficient than conventional binary tree multiplexing which offers $2 \log _{2} n$ scaling ratio. Table 1 shows the number of reactor cells addressable with $N$ air pressure inputs using the proposed method in comparison with some other multiplexing methods. 
Table 1. Number of cells addressable with $N$ pressure inputs using different multiplexing algorithms.

\begin{tabular}{lccc}
\hline \multirow{2}{*}{$\begin{array}{l}\text { Number of } \\
\text { pressure }\end{array}$} & \multicolumn{3}{c}{ Number of cells addressable } \\
\cline { 2 - 4 } inputs, $N$ & Proposed, & Binary tree [14], & $\begin{array}{l}\text { Square array [15], } \\
(N / 2)^{2}\end{array}$ \\
\hline 6 & 20 & 8 & 9 \\
8 & 70 & 16 & 16 \\
12 & 924 & 24 & 36 \\
16 & 12870 & 256 & 64 \\
\hline
\end{tabular}

\subsection{Fluidic model}

The basic structural components of the array platform are microvalves, fluidic channels and microreactors. Pneumatic actuation offers almost instant valve response, and the theoretical modeling in this paper is focused on the relationship between flow rates and applied pressures.

The flow rates through the microvalve are regulated by the pressure difference across the valve seat and the difference between the air pressure applied above the valve membrane and the hydraulic pressure under the valve membrane. Recently H Takao and M Ishida have found a good similarity between the $I-V$ characteristics of the $p$-MOSFET and the fluidic behaviors of this transistor-type microvalve [1].

For an unsaturated $p$-MOSFET transistor [16], the sourcedrain current is expressed as

$$
I=K\left[\left(V_{\mathrm{sg}}-\left|V_{\mathrm{th}}\right|\right) V_{\mathrm{sd}}-\frac{V_{\mathrm{sd}}^{2}}{2}\right],
$$

where $K$ is the transistor gain, a device-dependent constant. $V_{\mathrm{gs}}\left(V_{\mathrm{sg}}\right)$ is the difference between the gate voltage and source voltage, $V_{\text {th }}$ is the threshold voltage and $V_{\mathrm{ds}}\left(V_{\mathrm{sd}}\right)$ is the difference between the drain voltage and source voltage.

The analogous expression is then applied to the transistortype microvalve

$$
Q=F\left[\left(P_{\mathrm{sg}}-P_{\mathrm{th}}\right) P_{\mathrm{sd}}-\frac{P_{\mathrm{sd}}^{2}}{2}\right],
$$

where $Q$ is the volumetric flow rates. $F$ is a constant determined by valve seat dimensions, valve membrane properties and the viscosity of the fluid. $P_{\mathrm{sg}}$ denotes $P_{\text {source }}-$ $P_{\text {gate }}$, the pressure at the source side of the valve seats minus the air pressure above the valve membrane (gate), and $P_{\text {sd }}=$ $P_{\text {source }}-P_{\text {drain }} . P_{\text {th }}$ is the initial hydraulic pressure required to open the microvalve, which will be determined experimentally.

The fluidic channels and microreactors can be modeled as rectangular tubes. The small dimension in the microfluidic domain usually results in a small Reynolds number and thus laminar type flow. The steady flow of a Newtonian fluid in a circular tube follows Poiseuille's law:

$$
Q=-\frac{\pi R^{4} \Delta P}{8 \mu \mathrm{L}} \approx-\pi\left(\frac{w d}{w+d}\right)^{4} \frac{\Delta P}{8 \mu \mathrm{L}}
$$

For the rectangular tube the original radius is replaced by $w d /(w+d) . w$ and $d$ are the width and depth of the channel, $L$ is the length and $\mu$ is the viscosity of the fluid. It can be seen that the flow rate is proportional to the total pressure drop across the channel, which is analogous to the current-voltage curve of a simple resistor.

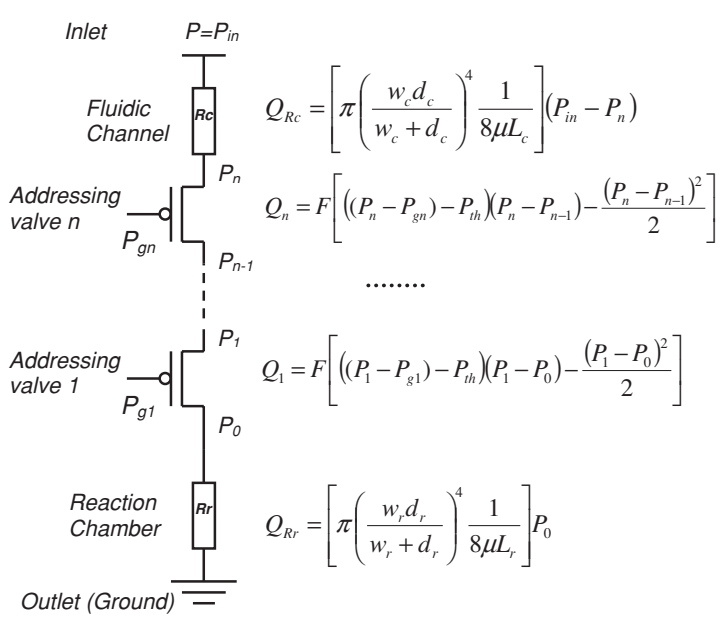

Figure 4. The equivalent circuit model of a reactor unit including reaction chamber, addressing microvalves and the fluidic channel.

Therefore an individual reactor unit including reaction chamber, fluidic channel and addressing microvalves is modeled as a serial connection of resistors and $p$-MOSFET transistors, which is illustrated in figure 4.

The flow rate (current) through each component can be calculated from its own structural parameters and the pressure drop over it, and these individually calculated flow rates have to be identical. Therefore an equation set can be obtained with equal numbers of unknowns and equations:

$Q_{\mathrm{Rr}}=\left[\pi\left(\frac{w_{\mathrm{r}} d_{\mathrm{r}}}{w_{\mathrm{r}}+d_{\mathrm{r}}}\right)^{4} \frac{1}{8 \mu \mathrm{L}_{\mathrm{r}}}\right] P_{0}$
$Q_{1}=F\left[\left(\left(P_{1}-P_{\mathrm{g} 1}\right)-P_{\mathrm{th}}\right)\left(P_{1}-P_{0}\right)-\frac{\left(P_{1}-P_{0}\right)^{2}}{2}\right]$

$Q_{\mathrm{n}}=F\left[\left(\left(P_{\mathrm{n}}-P_{\mathrm{gn}}\right)-P_{\mathrm{th}}\right)\left(P_{\mathrm{n}}-P_{\mathrm{n} 1}\right)-\frac{\left(P_{\mathrm{n}}-P_{\mathrm{n} 1}\right)^{2}}{2}\right](7 c)$

$Q_{\mathrm{Rc}}=\left[\pi\left(\frac{w_{\mathrm{c}} d_{\mathrm{c}}}{w_{\mathrm{c}}+d_{\mathrm{c}}}\right)^{4} \frac{1}{8 \mu \mathrm{L}_{\mathrm{c}}}\right]\left(P_{\mathrm{in}}-P_{\mathrm{n}}\right)$

$Q_{\mathrm{r}}=Q_{1}=\cdots=Q_{\mathrm{n}}=Q_{\mathrm{c}}$.

Finally the flow rate in the network can be solved as long as the inlet fluidic pressure and the air pressure for valve control are given. The validity and accuracy of this model will be examined using the experimental data in this paper. Modeling microfluidic components as simple electronic components and establishing an equivalent circuit, if successful, offers an intuitive and convenient tool to understand the flow/pressure distribution in fluidic networks, which is very helpful for the design of complex microfluidic systems.

\section{Methods and materials}

\subsection{Device fabrication and assembly}

The fabrication involved individual processing of a silicon wafer for fluidic channels and microreactors, a parylene/PDMS double-layer valve membrane and a PDMS cap for air pressure supply channels, as well as the assembly of all three components. The process flow is illustrated in 


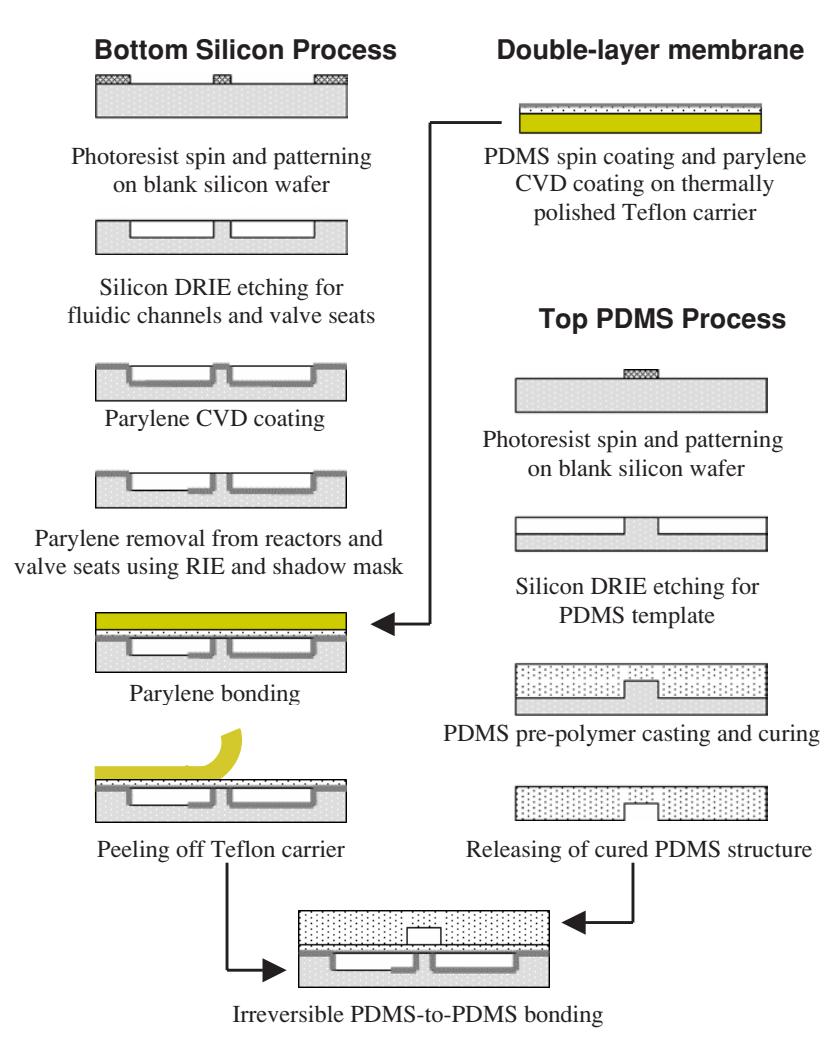

Figure 5. Process flow for device fabrication.

figure 5. First, a $20 \mu \mathrm{m}$ thick AZ9260 photoresist (Clariant Corp., USA) was spin coated on a 4 inch silicon wafer (Silicon Valley Microelectronics Inc., USA) and patterned using mask aligner (EVG420, EV Group, Austria). The UV-exposed photoresist was removed in 1:3 AZ400K (Clariant Corp., USA): $\mathrm{H}_{2} \mathrm{O}$ solution. Next $350 \mu \mathrm{m}$ deep microreactors and fluidic channels were formed using deep RIE silicon etching (STS deep silicon etcher, Surface Technology Systems, UK). The process parameters were $160 \mathrm{sccm} \mathrm{SF}_{6}, 800 \mathrm{~W} \mathrm{RF}$ power for the etch cycle and $85 \mathrm{sccm} \mathrm{C}_{4} \mathrm{~F}_{8}, 600 \mathrm{~W} \mathrm{RF}$ power for the passivation cycle. After STS etching the wafer was soaked in PRS2000 (JT Baker Inc., USA) to strip the remaining photoresist and cleaned using piranha solution (mixture of $\mathrm{H}_{2} \mathrm{O}_{2}$ and $\mathrm{H}_{2} \mathrm{SO}_{4}$ ).

Next a $2 \mu \mathrm{m}$ parylene-C film was uniformly deposited on the silicon wafer using chemical vapor deposition (PDS 2010 LABCOTER 2, Special Coating Systems, USA). The entire process includes sublimation of the solid parylene dimer (120-150 ${ }^{\circ} \mathrm{C}, 1.0$ Torr), pyrolysis of the dimer into monomers $\left(\sim 700{ }^{\circ} \mathrm{C}, \sim 0.5\right.$ Torr $)$ and final deposition of the parylene monomers $\left(25{ }^{\circ} \mathrm{C}, 0.1\right.$ Torr $)$. A 174 promoter was applied to improve the adhesion between silicon and parylene film. The parylene coating needs to be removed from the valve seats to prevent bonding with the valve membrane, and also from the reactor surface to expose the silicon substrate for DNA immobilization and many other biochemical reactions. The patterning of parylene film was achieved using oxygen plasma RIE (Semigroup Reactive Ion Etcher, the Semi Group Inc., USA) and a silicon wafer shadow mask. The process condition was $100 \mathrm{sccm} \mathrm{O}_{2}, 100$ mTorr pressure and $150 \mathrm{~W}$ $\mathrm{RF}$ power with an etching rate of $0.2 \mu \mathrm{m} \mathrm{min}^{-1}$.
The transfer of the parylene valve membrane was conducted using a Teflon carrier. A raw Teflon sheet ordered from a commercial vendor (McMaster-Carr, USA) was first sandwiched between two silicon wafers with a 4 MPa pressure applied and heated to $300{ }^{\circ} \mathrm{C}$, which is above Teflon's glass transition temperature. The Teflon surface was thus polished and became very smooth. This Teflon carrier was then spin coated with the mixture (10:1 weight ratio) of PDMS and its curing agent (Sylgard ${ }^{\circledR} 184$, Dow Corning Co., USA) at $3 \mathrm{krpm}$ and baked for $1 \mathrm{~h}$ at $100{ }^{\circ} \mathrm{C}$ to form a $60 \mu \mathrm{m}$ PDMS layer, which was followed by CVD coating of another $2 \mu \mathrm{m}$ parylene thin film. The Teflon/PDMS/parylene sheet was next brought into contact with the previously processed silicon wafer and both pieces were placed in a manual hot press tool. Heat $\left(160{ }^{\circ} \mathrm{C}\right)$ and pressure $(1$ bar) were applied to achieve the bonding between the parylene-coated silicon wafer and parylene/PDMS double-layer thin film. The bonding mechanism is that, above the glass transition temperature $\left(\sim 109{ }^{\circ} \mathrm{C}\right)$, heat enables the polymer chains in two adjacent parylene films to move around and eventually get entangled with each other [12, 13]. After cooling down the fused parylene films serve as an intermediate adhesive layer, resulting in very strong and irreversible bonding of the two substrates with parylene coating. This technique is particularly suitable for the fabrication of the proposed device because parylene serves as not only the valve membrane but also the bonding adhesive. After parylene bonding, the semiflexible Teflon carrier was carefully peeled away due to the weak adhesion between Teflon and PDMS; this left on the etched silicon wafer a flat and undamaged parylene/PDMS double-layer membrane which covers all the microfluidic channels and microreactors. The membrane and valve seats were still kept apart because parylene on valve seats was previously removed and there is no bonding between parylene thin film and the bare silicon substrate. Besides the Teflon sheet we also tried a silicon wafer, PDMS sheet and Mylar sheet as the carriers for membrane transfer. The results will be given in the following section.

The PDMS cap containing air pressure supply channels was molded using a silicon master fabricated by deep RIE silicon etching. Prior to PDMS casting, the silicon master was exposed to trimethylchlorosilane vapor for $10 \mathrm{~min}$ for future mold release. Then, the liquid PDMS prepolymer (10:1 mixing ratio) was poured onto the silicon master and left at room temperature for at least $24 \mathrm{~h}$, and the cured PDMS was peeled off from the silicon mold to complete the air cap fabrication. Room temperature curing is necessary in this process because it maintains highdimensional fidelity. Regular thermal curing $\left(65^{\circ} \mathrm{C}\right.$ or higher) produces considerable size shrinkage from designed values (master dimensions) after peeling off the PDMS structure from the master, which is not allowed for a high density array because of the mismatch between the air cap and fluidic structures on silicon.

Next the PDMS cap and the silicon microchip covered by transferred parylene/PDMS membrane were cleaned using isopropyl alcohol and treated in oxygen plasma for $30 \mathrm{~s}$. The two pieces were then aligned using a microscope and quickly put into contact. Irreversible bonding was achieved immediately because the contacting surfaces of both pieces 


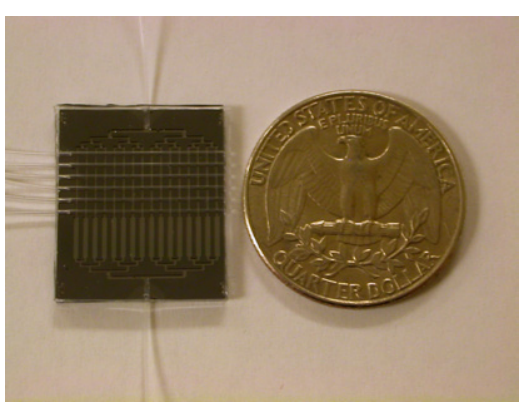

Figure 6. A fabricated microvalve/reactor array platform with tubing connected.

were PDMS. Heating of the assembled structure in an oven at $80{ }^{\circ} \mathrm{C}$ could further strengthen the bonding.

Finally a side-feeding configuration was used to make the fluidic interface between the microchip and external sources. The deep trenches etched on silicon were covered by the top PDMS cap and formed closed channels connecting to the microreactors on the device. The ends of these channels were opened on the side of the microchip and allowed the insertion of $400 \mu \mathrm{m}$ OD PEEK tubes (Upchurch Scientific, USA). Epoxy glue (5 Minute ${ }^{\circledR}$ Epoxy, Devcon, USA) sealing was applied to the channel openings after tubing insertion. This is a very convenient way to build the fluidic interface, and it eliminates the use of external fluidic connectors or couplers which are relatively expensive. Also, there are no visual obstacles on the upper surface of the device, which greatly facilitates real-time monitoring during device operation. The fabricated and assembled microarray chip with tubing insertion is illustrated in figure 6 .

\subsection{Fluidic tests}

We designed experiments to explore the sealing ability of closed valve and the effect of the inlet water pressure on the fluidic flow rate in the valve-opened channel. Also, different fluidic channels with various numbers of opened microvalves and various valve seat dimensions were fabricated and individually tested. Experimental results were compared with the theoretical prediction using the electronic equivalent model to evaluate its accuracy.

The pumping for flow injection was simply achieved by introducing compressed air into a DI water container to provide water flow with a constant pressure. Commercial solenoid valves (ETO-3M, Clippard Minimatics, USA) were used to switch on/off the air pressure supply to the pressure channel for the microvalves. The flow rates were recorded by collecting the outlet flow over a certain period and weighing the collected liquid on an analytic balance. All runs were repeated at least three times and the average values were taken.

\subsection{Chemical tests}

In order to test the chemical resistance of the parylene-based microvalve, we chose the major reagents used for liquidphase DNA synthesis on solid supports. They are acetonitrile, capping solution (acetic anhydride and tetrahydrofuran), deblock solution (dichloroacetic acid and dichloromethane) and oxidizer solution (iodine, pyridine, tetrahydrofuran and water). These chemicals included organic solvents, acid, base and oxidizer, which are typical representatives of aggressive chemicals involved in chemical and biological reactions. Also, synthesis of oligonucleotide libraries on microchip is one of the most important applications we are envisioning for the presented array platform. It must demonstrate good operation stability when handling the above chemicals.

A commercial nucleic acid synthesis system (Expedite Model 8909, Millipore Corp., USA) was used to pump each of the testing chemicals into the fluidic channel containing opened microvalves. The pumping was conducted as a sequence of pulses with constant hydraulic pressure (6 psi). The duration time of each pulse is also constant. Therefore the flow rate through the fluidic channel should remain unchanged from pulse to pulse if the microvalve is not affected by the chemicals passing through it. In contrast, the flow rate is expected to decrease with time if the microvalve shows swelling, which is a typical behavior for many polymeric materials exposed to organic solvents. To measure the flow rates we collected the liquid from the fluidic channel outlet in each pulse and weighted it using an analytic balance, and the amount of collected liquid will be proportional to the average flow rate during the pulse. As a comparison, another type of microvalve was made by replacing the parylene valve membrane with PDMS. The valve structure and dimensions remained unchanged. It has been reported that PDMS swells considerably in many solvents. The above chemical resistance tests were also performed on this PDMS-based microvalve to examine how swelling affects the valve performance.

\subsection{On-chip synthesis of DNA oligonucleotide using microvalve array addressing}

The synthesis process started with the derivatization of the microreactors using a $1 \mathrm{wt} \%$ solution of $\mathrm{N}$-(3-triethoxysilylpropyl)-4-hydrooxybutyramide linker in ethanol, which was an overnight reaction at room temperature. During this step all of the valves in the array were open so that bare silicon oxide surfaces in all microreactors were grafted with linker molecules. The fluidic channels were coated with parylene film. The hydrophobic parylene does not develop the surface hydroxyl group, which is required for the covalent binding with linker molecules. Also, unlike porous PDMS, parylene demonstrates little non-specific adsorption of other molecules [7, 17]. Therefore the linker molecules were expected to be immobilized only in microreactors. After derivatization, the microchip was rinsed thoroughly with $95 \%$ ethanol and cured under nitrogen gas at $100{ }^{\circ} \mathrm{C}$ for $1 \mathrm{~h}$. It was then connected to an Expedite 8909 DNA synthesizer using PEEK tubing and connectors (Upchurch Scientific, Oak Harbor, WA). The synthesizer was programmed to deliver reagents to the microchip for DNA synthesis. The microvalve array was actuated during synthesis to direct the growth of oligonucleotides only in selected microreactors. The synthesis was conducted using standard protocol of phosphoramidite chemistry, which involves repeating cycles of deblocking, coupling, capping and oxidation. The fluidic parameters on the synthesizer originally set for standard CPG columns were also modified according to the microchip volume and the flow rates through it. Oligonucleotides 
with a 30-mer sequence and a T15-mer spacer, 5'AGAAGTCTAATATCGACGAAGCATTATTTCtttttttttttttt$3^{\prime}$, were synthesized in target reactors selected by the microvalve array. The synthesis was followed by extensive rinse with ethanol and then deprotection of the synthesized oligonucleotides for hybridization purpose using EDA/ ethanol (anhydrous, 1:1 v/v) mixture at room temperature for $2 \mathrm{~h}$. Finally the microchip was thoroughly rinsed with ethanol and a sodium phosphate buffer, and then was ready for sequence verification.

\subsection{Validation of the synthesized oligonucleotide sequences by a hybridization experiment}

$1 \mu \mathrm{g}$ Cy-5 labeled target DNA with the complementary sequence was dissolved in $100 \mu \mathrm{l} 6 \times \mathrm{SSPE}$ buffer $(1 \mathrm{M} \mathrm{NaCl}$, $66 \mathrm{mM}$ sodium phosphate, $6 \mathrm{mM}$ EDTA, $\mathrm{pH}$ 7.4) and the solution was injected into the microchip for hybridization. The entire valve array was opened again; therefore the hybridization solution entered all the microreactors, including those not selected for the growth of oligonucleotides. The hybridization reaction took overnight at room temperature. After that, the microchip was washed with $6 \times$ SSPE and $1 \times$ SSPE solution in turn. Finally the fluorescent image was captured using a DNA microarray scanner (Genepix 4000B, Axon Instruments, CA).

\section{Results and discussions}

\subsection{The membrane transfer process}

The most critical step in the fabrication was the transfer of the thin parylene membrane onto the bottom silicon wafer containing previously etched microfluidic structures. The transfer mechanism is the thermal bonding of a parylene film deposited on a flat substrate to a parylene-coated target substrate followed by the removal of the original substrate (carrier). Four different substrates, a silicon wafer, PDMS slab, Mylar sheet and Teflon sheet, were tested as the transfer carriers. From the results we found the silicon wafer was not a capable carrier. The parylene membrane or even the silicon wafer always broke when separating the silicon carrier from the structure wafer due to the brittle nature of silicon. Although alternative methods are available such as applying dissolvable sacrificial photoresist between parylene and the silicon carrier, or etching away the entire silicon carrier, both of them are relatively labor intensive and time consuming. Therefore employing a flexible transfer carrier appears as a better approach.

Using a wafer-shaped PDMS slab or Mylar sheet as the carriers, we achieved the transfer of an undamaged parylene membrane onto the silicon microfluidic wafer. However, we found that the transferred membrane was significantly concaved above all fluidic channels and microreactors, which resulted in a non-operational microvalve array because pressurizing air could leak between adjacent channels and cause crosstalk among different microvalves in the array (shown in figure 7). The reason was that the PDMS slab and Mylar sheet were too soft and deformed considerably under the applied pressure during parylene bonding. The parylene film coated on the PDMS slab or Mylar sheet was a thermoplastic
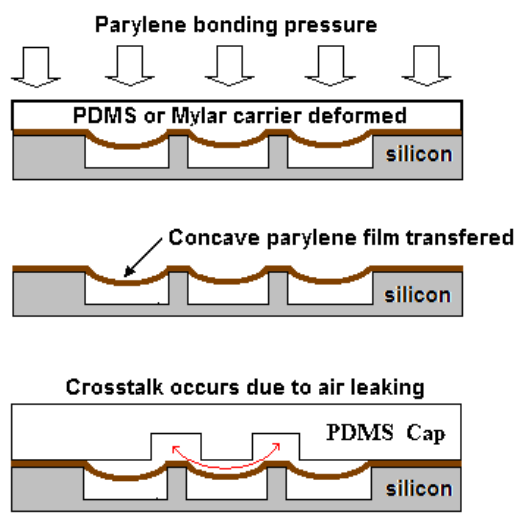

Figure 7. Concave parylene membrane and valve array crosstalk caused by inappropriate membrane transfer carriers.

polymer and adapted to the surface profile of the deformed carrier. After the bonded device cooled down and pressure was removed, the thermoplastic parylene film could not restore to its original shape and remained concave.

The Teflon sheet turned out to be the best candidate because it is flexible enough to be bent and removed from transferred parylene membrane like a zipper, but stiff enough not to noticeably deform during parylene bonding. The only problem left was the rough surface of the commercially available Teflon sheets. We therefore performed thermal polishing as described before reducing the surface roughness from $2 \mu \mathrm{m}$ to $0.2 \mu \mathrm{m}$. This enabled the successful wafer-level transfer of a flat, smooth and undamaged parylene membrane. The surface profiles of the transferred parylene membranes across the microfluidic channels were measured using Dektak $6 \mathrm{M}$ surface profiler. The Mylar carrier-transferred parylene membrane exhibited a concave depth of $65 \mu \mathrm{m}$ over the fluidic channel, while the value for the Teflon-transferred membrane was merely a few microns.

\subsection{Fluidic properties}

The single valve functionality was tested by measuring the flow rates through a fluidic channel containing one microvalve under different sealing pressures and inlet pressures. The channel was $2 \mathrm{~cm}$ long, $350 \mu \mathrm{m}$ deep and $550 \mu \mathrm{m}$ wide. First a 15 psi pressure was applied to close the microvalve and block the fluidic channel; then the flow inlet pressure was increased to examine the valve sealing ability. There was no leakage detected until the inlet pressure increased beyond $20 \mathrm{psi}$, which was already higher than the sealing pressure and indicated a 'threshold' pressure difference for opening the transistor-type valve. This result shows that the microvalve provides excellent leakage-free sealing against the inlet (source) pressure as long as it does not exceed the sealing (gate) pressure by the 'threshold' value $\left(P_{\mathrm{sg}}<P_{\mathrm{th}}\right)$.

The water flow rates through the valve-opened channel $\left(P_{\text {gate }}=0\right)$ under different inlet pressures are shown in figure 8 . All the values are the average of three or more repeated runs under the same condition. At a very small inlet pressure no flow is observed. The flow rate starts to be noticeable at a 3 psi inlet pressure and increases rapidly after that. The threshold pressure $P_{\text {th }}$ is therefore determined to be 3 psi, which is the 


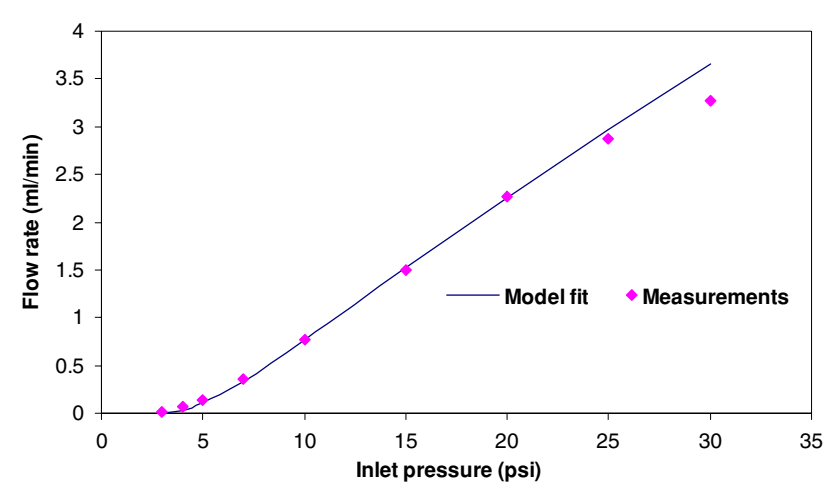

Figure 8. Water flow rate through a fluidic channel containing one opened microvalve $\left(P_{\text {gate }}=0\right)$ under different inlet pressures $\left(P_{\text {inlet }}\right)$, and data fit using the equivalent circuit model.

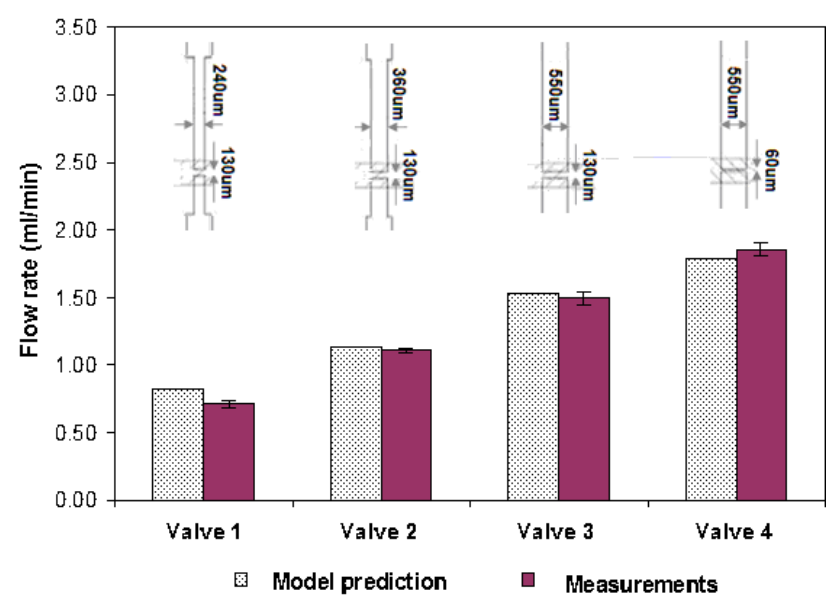

Figure 9. Water flow rates through fluidic channels containing one opened microvalve with various valve seat dimensions $\left(P_{\text {gate }}=0\right.$, $\left.P_{\text {inlet }}=15 \mathrm{psi}\right)$, and theoretical predication using the equivalent circuit model.

minimum force required to overcome the initial stiction and lift up the parylene valve membrane. In the moderate pressure region (7-25 psi), there is a nearly linear relationship between the inlet pressure and flow rate. After the inlet pressure reaches a higher range $(>25 \mathrm{psi})$, the increase in the flow rate slows down, and the epoxy sealing between the tubing and plastics/PDMS isprone to leak. Also shown in figure 8 is the resistor-transistor model prediction, which provides an excellent fit to the measurements for inlet pressures lower than 25 psi. The value of the transistor gain, $F$, for the model was determined as $0.085 \mathrm{ml} \mathrm{min}{ }^{-1} \mathrm{psi}^{-2}$. At a higher pressure range ( $>30 \mathrm{psi}$ ), the discrepancy between the model prediction and real measurements starts to be significant. This may be explained by the fact that the mathematical correlation between the valve membrane deflection and gate pressure becomes highly nonlinear for large deflections, and the simple transistor approximation is no longer reliable. Also, the likely leaking of epoxy seals under high pressures reduces the effective pressure drops and valve-opened flow rates. Nevertheless, the pressures involved in most microfluidic-based applications are lower than $20 \mathrm{psi}$, and the resistor-transistor model is quite accurate in this moderate pressure range.

Figure 9 shows the water flow rates through valve-opened channels with different valve seat dimensions under a 15 psi inlet pressure. As can be seen, both enlarging valve seat width and reducing valve seat length increase the flow rate significantly. We know that for the $p$-MOSFET the transistor gain $K=\mu_{\mathrm{p}} C_{\mathrm{ox}}(W / L)$ [16]. $\mu_{\mathrm{p}}$ is the charge mobility, $C_{\mathrm{ox}}$ is the capacitance of the gate oxide and $W$ and $L$ are the width and length of the PMOS channel respectively. We expect a similar expression for the gain $(F)$ of the transistor-type microvalve $F=\mu^{-1} C_{\mathrm{m}}(W / L)$, where $\mu$ is the viscosity of the fluid and $\mu^{-1}$ represents the 'fluid mobility'. Deionized water ( $\mu=$ $0.894 \mathrm{cp}$ at $25^{\circ} \mathrm{C}$ ) was used for all the fluidic tests in this work. $C_{\mathrm{m}}$ is the parameter determined by the valve membrane area, thickness and Young's modulus. Using a thin and soft valve membrane results in a larger $C_{\mathrm{m}}$ and therefore larger $F$. From the previous results $\left(F=0.085 \mathrm{ml} \mathrm{min}{ }^{-1} \mathrm{psi}^{-2}\right.$ for a $550 \mu \mathrm{m}$ wide, $130 \mu \mathrm{m}$ long valve seat), we set $\mu^{-1} C_{\mathrm{m}}=$ 0.02017 for water flow in our particular device. Then the resistor-transistor model was applied to calculate the flow rates using the rule of $F=0.02017(W / L)$ for different valve seats and adjusted 'resistance' for the fluidic channels with changing width. The results are shown in figure 9 and compared with the measurements. The theoretical calculation provides fairly close estimation for the real flow rates (error $<5 \%$ for valves 2,3 and 4 ), which further substantiates the model validity.

Wider and shorter valve seats are preferred for high valveopened flow rates. The width of valve seat is limited by space and integration density. Very short valve seat can be readily fabricated on silicon using high aspect ratio deep RIE etching, but is relatively hard to be implemented on glass substrates due to the undercutting during wet etching of glass.

Both experiments and model estimation show that the transistor-type microvalve introduces small flow impedance which is less than that of the fluidic channels and tubing connections. The $2 \mathrm{~cm}$ long, $550 \mu \mathrm{m}$ wide and $350 \mu \mathrm{m}$ deep fluidic channels with an opened valve exhibited a water flow rate of $2.87 \mathrm{ml} \mathrm{min}^{-1}$ at a $25 \mathrm{psi}$ inlet pressure, which is significantly higher than most of the reported microvalves. This small flow impedance device can realize the control over a large dynamic range of flow rates, which is a quite desirable feature for high-throughput chemical and biomedical applications.

As described previously, when the number of microreactors increases more microvalves need to be distributed in a fluidic channel to address the corresponding microreactor, and the flow impedance introduced by multiple in-channel microvalves becomes a possible concern. We therefore measured the valve-opened flow rates through a series of channels containing $0,1,2$ and 3 microvalves respectively, and the results are shown in figure 10.

The reference channel (no valve) exhibited a water flow rate of $2.1 \mathrm{ml} \mathrm{min}^{-1}$ at a $15 \mathrm{psi}$ inlet pressure. This result was inputted to the model to calibrate the total flow resistance $R$ caused by the fluidic channel, reactor, tubing and geometry factors which are modeled as simple resistors.

The introduction of the first microvalve reduced the flow rate by $29 \%$, but the second and third microvalves only further reduced it by $16 \%$ and $3 \%$ respectively. The calculation using the circuit equivalent model also indicates a similar trend with significantly damped rate of decrease in the flow rate. The reason is that the fluidic channels instead of the opened 


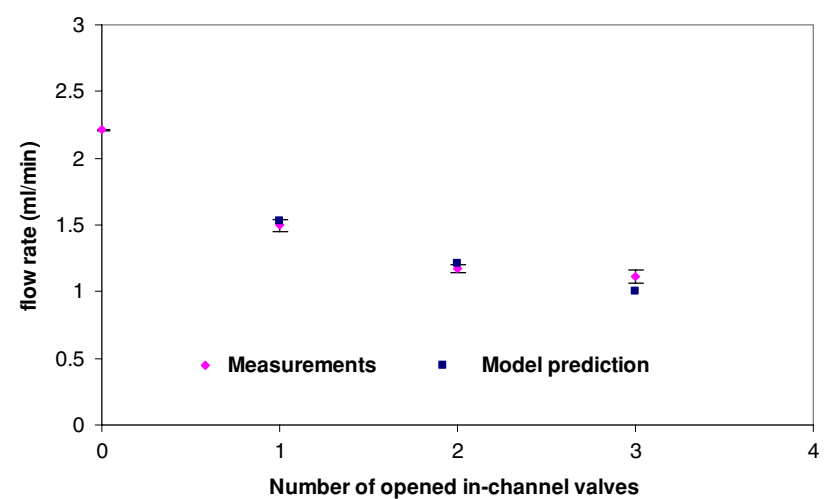

Figure 10. Water flow rates through fluidic channels containing an increasing number of opened microvalves $\left(P_{\text {gate }}=0, P_{\text {inlet }}=15 \mathrm{psi}\right)$, and theoretical predication using the equivalent circuit model.

microvalves make the dominant contribution to the overall flow impedance; also, more total pressure drop is distributed over the valve series as more opened microvalves are added to a fluidic channel and most of the source-gate pressure differences are still maintained. All of these mitigate the increase of flow impedance and the final flow rates will still be tolerable. The operation of the high density microreactor array with multiple in-channel addressing valves is therefore feasible.

\subsection{Chemical compatibility}

During the chemical resistance tests, the flow rates of different chemicals through the microfluidic channel containing an opened microvalve were monitored for three consequent pulses. Each pulse lasted about $1 \mathrm{~min}$, and the interval between two pulses was also $1 \mathrm{~min}$. For the reference chip with the pure PDMS membrane, significant decreases of flow rates were observed from pulse to pulse in a DNA monomer capping solution (acetic anhydride and tetrahydrofuran) and monomer deblock solution (dichloroacetic acid and dichloromethane). The capping reagent and deblock reagent are mixtures of a few chemicals, but we believe that the solvents (tetrahydrofuran and dichloromethane) made major contributions to the swelling of PDMS. The results are given in figure 11. For each chemical the flow rates of pulses 1,2, 3 were normalized by setting the flow rate of pulse 1 as the standard. The most severe degradation of the PDMS microvalve occurs in dichloromethane, which caused almost $90 \%$ flow rate drop in a few minutes.

Next we conducted the same experiments on the parylenebased device. The results are also given in figure 11 and show superior performance. There was no noticeable drop of flow rate for all the DNA synthesis chemicals. Very stable flows were obtained from pulse to pulse, which demonstrates excellent chemical resistance of the proposed microarray platform with the parylene membrane surface.

\subsection{Addressed synthesis of DNA oligonucleotides on the microvalve/reactor platform}

Synthesis of 45-mer oligonucleotide was conducted in target microreactors using microvalve array addressing. The flow (a) DNA capping solution (acetic anhydride + tetrahydrofuran)

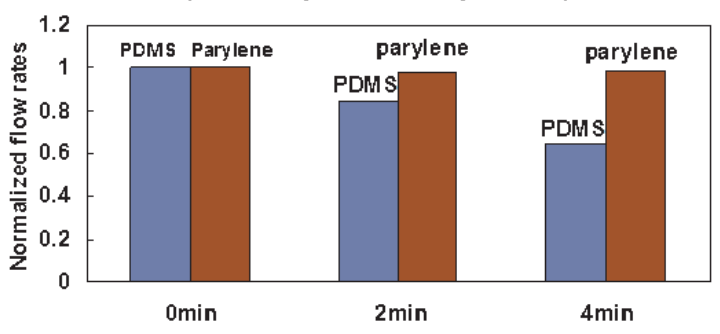

(b) DNA deblocking solution (dichloroacetic acid + dichloromethane)

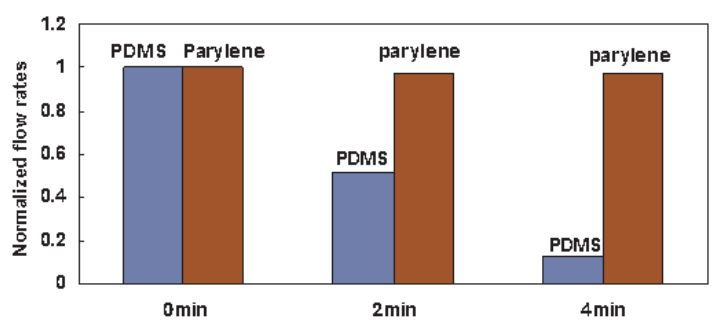

Figure 11. (a) Normalized flow rates of the DNA capping solution (acetic anhydride + tetrahydrofuran) through opened PDMS valve and parylene valve. (b) Normalized flow rates of the DNA deblock solution (dichloroacetic acid and dichloromethane) through opened PDMS valve and parylene valve.

rate through the microchip platform was monitored by measuring the volume of collected outlet flow after every five cycles during the synthesis. The flow remained stable through the entire synthesis process $(\sim 4 \mathrm{~h}$ with $\sim 120$ opening and closing cycles with four different chemical streams) for the parylene-coated PDMS valves except for the small (10\%) fluctuation caused by the synthesizer machine. The reference PDMS device showed the rapidly decreasing flow rate and was eventually blocked completely during the synthesis even before the completion of $10 \%$ of the cycles.

In this experiment air pressure was supplied to the second air trench from the left and closed the top two channels and bottom channel because all of them had one microvalve in this vertical column. All of the microvalves controlling the three channels in the middle were still open and oligonucleotides were synthesized in corresponding reactors. After synthesis, all microvalves were opened and Cy-5 hybridization reagents were injected into all the microreactors. Figure 12(a) shows the fluorescent image after an overnight hybridization reaction and subsequent buffer wash. In the figure, the three reactors with opened microvalves exhibit strong fluorescence, and the other three reactors are completely dark even though they were exposed to the fluorescent hybridization solution as well. These results illustrate reliable and leakage-free microvalve array addressing. Also, there is a strong contrast between the bright reactors with a bare silicon oxide surface and dark fluidic channels with a parylene-coated surface, which shows that parylene is an excellent surface passivation material. It exhibits not only the superior chemical resistance, but also little adsorption of other molecules. The weak signal on parylene film mostly comes from the intrinsic background fluorescence of parylene shown in figure 12(c). After subtracting this background from the image of figure 12(a), we found the linker attachment on parylene, which can cause 


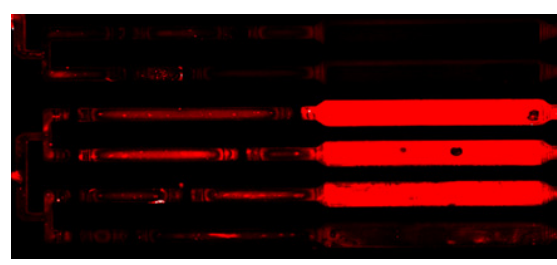

(a)

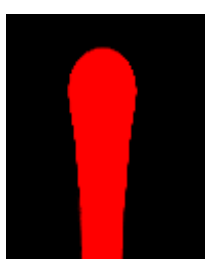

(b)

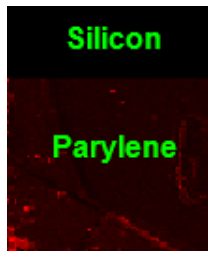

(c)

Figure 12. (a) Close-up view of the microchip fluorescent image after addressed synthesis of oligonucleotide in three target reactors (bright red) and hybridization with a Cy-5 labeled complementary sequence (the extremely weak fluorescence signal in the dark regions comes from intrinsic parylene background noise). (b) Control experiment (fluorescence of oligonucleotides synthesized and hybridized using the same recipe in a plain silicon reactor without microvalves). (c) Background fluorescence of parylene thin film deposited on the silicon substrate without exposure to any chemicals.

additional fluorescence, is trivial. In addition, the fluorescent intensities in these three microreactors appear identical and homogenous, indicating uniform flow distribution into all addressed microreactors.

To evaluate the synthesis efficiency of this parylene microvalve-controlled microreactor, we also conducted a control experiment in which the same oligonucleotides were synthesized and hybridized using the same recipe in a plain silicon reactor without the microvalve structure and parylene surfaces, and the result is illustrated in figure $12(b)$. It can be seen that parylene appears as a safe material for the reactions and the introduction of parylene microvalve does not significantly affect the synthesis efficiency. The average oligonucleotide fluorescence intensity in the parylene microvalve-controlled reactor after the synthesis of a 30mer sequence (figure $12(a)$ ) is $\sim 84 \%$ of that in the plain silicon reactor (figure $12(b)$ ). Since the actual synthesis process involved 30 different monomer addition steps, in order for the microvalve-based system to give a final signal that is $84 \%$ of the standard synthesis system, the microvalve synthesis yield during each step has to be $99.5 \%$ identical to the standard synthesis yield $\left(0.995^{30}=0.84\right)$. This yield is quite satisfactory for general applications. We can therefore conclude that addressed synthesis of DNA oligonucleotides with proper sequences using the integrated microvalve/reactor array platform is successful.

Future work based on this array platform includes the production of oligonucleotide libraries for large-scale artificial gene synthesis. If the microvalve array is controlled by a computer to deliver different DNA monomers to each reactor during each synthesis cycle, we can synthesize an array of different oligonucleotide sequences (oligonucleotide library) in parallel on the platform. After purification, the pool of synthesized sequences can be further assembled to much longer sequences using ligation or polymerase assembly multiplexing (PAM) reaction. This is a rapid and low-cost approach to synthesize large artificial genes which will be useful for gene expression research, combinatorial biology and gene therapy applications.

Currently there are other techniques available to produce DNA microarrays such as photo-directed parallel synthesis [18] and electrolytic acid/base-aided synthesis [19]. They all rely on specifically developed photo- and electrochemistry for oligonucleotide reaction only, and the amounts of produced oligonucleotides are quite small due to the limited surface areas. The integrated microfluidic circuit technology developed by S Quake and his co-workers offers precise manipulation of many discrete samples and reagents in an array and is also successfully used for multi-step reactions/synthesis [20-23]. However, the unsatisfactory solvent compatibility of the PDMS structure involved in this technology seriously limits the variety of chemical reactions that can be executed [23]. The chemical-resistant microvalve/reactor array presented in this work provides reliable physical addressing, multiplexing and isolation with significantly improved chemical compatibility. It is therefore a versatile tool which supports general chemical and biochemical reactions, and can be used for the combinatorial production of a good variety of chemicals or biological compounds. Also, the microreactor surface areas can be easily increased by orders of magnitude to achieve large production capacity by introducing porous microbeads or pillar-array structures into the microreactors.

\section{Conclusions}

In summary, we have developed and characterized a versatile microreactor platform featuring a novel microvalve array. The array control algorithm we proposed has a superior multiplexing efficiency and can achieve individual addressing of a large array of reactors with a very small number of signal inputs. The device fabrication combined the advantages of polymer processing and silicon bulk machining. The special membrane transfer process using parylene bonding and a polished Teflon carrier enables a wafer-level flat and smooth parylene membrane to be fabricated on etched structures. In addition, the deep silicon etching step involved in the fabrication allowed an easy side tubing connection to the microchip which requires little space, materials, and greatly facilitates the visual observation of the device operation.

The microvalve is leakproof when closed. In open status it exhibits low flow impedance and can therefore handle a much larger range of flow rates than most current microvalves. The equivalent circuit model constructed by treating the microvalves as PMOS transistors and the fluidic channels as simple resistors provided good agreements with the real measurements, and offers a useful tool for the design of complicated fluidic systems.

The array platform with the unique PDMS/parylene double-layer valve membrane demonstrated excellent compatibility with typical aggressive chemicals, which 
is an important improvement for conventional PDMSbased microfluidic devices. Addressed synthesis of DNA oligonucleotide on the microchip was successfully demonstrated and the results showed leakage-free valve array addressing, uniform flow distribution and excellent regional reaction selectivity. The presented microvalve/reactor array along with the robust array addressing strategy may function as a universal platform for multiplexed screening/discovery, reaction optimization, combinatorial synthesis of a variety of chemicals/biomacromolecules, and is particularly promising for those involving tough chemicals and wide dynamic range of flow rates.

\section{Acknowledgments}

The authors acknowledge the financial support for this work from The Defense Advanced Research Projects Agency and the National Institutes of Health. The authors would also like to thank Hanseup Kim for his assistance on the parylene bonding process.

\section{References}

[1] Takao H, Ishida M and Sawada K 2002 J. Microelectromech. Syst. 11 421-6

[2] Hosokawa K and Maeda R 2000 J. Micromech. Microeng. 10 415-20

[3] Grover W, Skelley A, Liu C, Lagally E and Mathies R 2003 Sensors Actuators B 89 315-23
[4] Baechi D, Buser R and Dual J 2002 Sensors Actuators A 95 77-83

[5] Unger M, Chou H, Thorsen T, Scherer A and Quake S 2000 Science 288 113-6

[6] Lee J, Park C and Whitesides G 2003 Anal. Chem. 75 6544-54

[7] Shin Y, Cho K, Lim S, Chung S, Park S, Chung C, Han D and Chang J 2003 J. Micromech. Microeng. 13 768-74

[8] Xie J, Yang X, Wang X Q and Tai Y C 2001 Proc. IEEE Micro Electro Mechanical Systems (Interlaken, Switzerland) pp 539-42

[9] Carlen E T and Mastrangelo C H 2002 J. Microelectromech. Syst. 11 408-20

[10] Xie J, Shih J and Tai Y C 2003 Proc. IEEE Micro Electro Mechanical Systems (Kyoto, Japan) pp 20-3

[11] Walsh K, Norville J and Tai Y C 2001 Proc. IEEE Micro Electro Mechanical Systems (Interlaken, Switzerland) pp 114-7

[12] Hanseup S and Najafi K 2003 12th Int. Conf. on Solid-State Sensors, Actuators, and Microsystems (Boston, MA)

[13] Noh H, Hesketh P J and Frye-Mason G C 2002 J. Micromech. Microeng. 11 718-25

[14] Thorsen T, Maerkl S and Quake S 2002 Science 298 580-4

[15] Pan J, VerLee D and Mehregany M 1997 Proc. Transducers' 97 (Chicago, IL) pp 817-20

[16] Gray P and Meyer R 1993 Analysis and Design of Analog Integrated Circuits 3rd edn (New York: Wiley)

[17] Pal R et al 2005 Lab Chip 5 1024-32

[18] www.affymetrix.com

[19] www.combimatrix.com

[20] www.fluidigm.com

[21] Kartalov E and Quake S 2004 Nucl. Acids Res. 32 2873-9

[22] Hong J, Studer V, Hang G, Anderson W and Quake S 2004 Nat. Biotechnol. 22 435-9

[23] Lee C et al 2005 Science 310 1793-6 